\title{
Electrically Controlled Liquid Crystal Plasmonic Metamaterials
}

\author{
O. Buchnev, ${ }^{1, *}$ J. Y. Ou, ${ }^{1}$ M. Kaczmarek, ${ }^{2}$ N. I. Zheludev, ${ }^{1}$ and V. A. Fedotov ${ }^{1}$ \\ 1 Optoelectronics Research Centre, University of Southampton, SO17 1BJ, UK \\ 2 School of Physics and Astronomy, University of Southampton, SO17 1BJ, UK
}

We experimentally demonstrate efficient electrical modulation and control of the near-IR response of plasmonic metamaterials loaded with liquid crystals (LC) by exploiting micro-scale volume and (for the first time) nano-scale in-plane LC-switching modes. We also show that in the resulting hybrid optical cell the metamaterial nano-structure can replace all three essential components of an LC device: (i) LC-alignment layer; (ii) transparent electrode and (iii) polarizer; making the hybrid cell more compact than the conventional LC devices and easy to integrate into plasmonic and nano-photonic circuits. The relative ease of on-demand engineering resonant bands (i.e. colours) and tailoring reflection/refraction phenomena in metamaterials is particularly appealing for application in high-resolution and emerging display technologies including near-to-eye and virtual retina displays, holographic and 3D imaging.

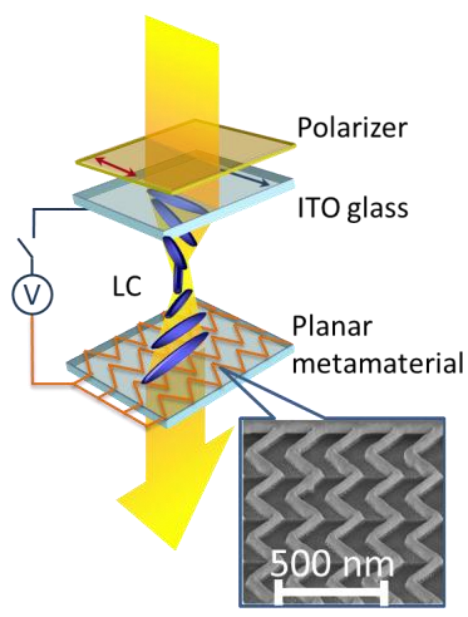

Design of a hybrid metamaterial-based liquid-crystal optical cell. Inset shows SEM micrograph of the fabricated metamaterial nano-structure.

The control of the metamaterial response using volume LC-switching was achieved by integrating metamaterial into a LC cell. It was comprised of a $15 \mu \mathrm{m}$ thick layer of nematic LC confined between the metamaterial and a transparent electrode coated with LC-alignment layer (see Figure). The structure of the metamaterial was formed by a continuous zig-zag wire nano-pattern that was milled by FIB in a 80 $\mathrm{nm}$ thick $\mathrm{Au}$ film deposited on a glass substrate. Direct contact between the liquid crystal and nano-structure provided anchoring and aligned LC molecules orthogonal with respect to the molecules at the transparent electrode leading to the twisted ordering. While metallic fabric of the metamaterial served as the second electrode, its polarization sensitive resonance determined optical response of the hybrid cell. In particular, the resonant polarization of incident light that would excite plasmons in the nano-structure at $1.5 \mu \mathrm{m}$ became non-resonant while propagating in the twisted cell and was transmitted. By applying electric field of up to $7 \mathrm{~V}$ we could reversibly destroy the cell's twisted state and reduce the metamaterial transmission by a factor of five.

The in-plane LC switching was demonstrated for a substrate-free negative (complimentary) version of $80 \mathrm{~nm}$ thick metamaterial nano-structure, which was suspended on $100 \mathrm{~nm}$ thick silicon-nitride bridges. The absence of the substrate material in the gaps between the zig-zag elements of the metamaterial network substantially reduced the anchoring of the LC-molecules, permitting their reorientation in the gaps under electric field applied in the plane of the nano-structure. The in-plane switching allowed us to control both the amplitude and spectral position of the metamaterial transmission band, and bring the operating voltage below $2 \mathrm{~V}$ by engaging LC layer near the metamaterial with the thickness of only few hundreds of nanometers.

\footnotetext{
* presenting author; E-mail: O.Buchnev@ soton.ac.uk
} 